\title{
ARRP-21, a Cyclic AMP-Regulated Phosphoprotein Enriched in Dopamine-Innervated Brain Regions. II. Immunocytochemical Localization in Rat Brain
}

\author{
C. C. Ouimet, ${ }^{a}$ H. C. Hemmings, Jr., ${ }^{b}$ and P. Greengard \\ Laboratory of Molecular and Cellular Neuroscience, The Rockefeller University, New York, New York 10021
}

\begin{abstract}
ARPP-21, a cAMP-regulated phosphoprotein, has been studied by immunocytochemistry to determine its cellular and regional distribution in rat brain. This study demonstrates that ARPP-21 immunoreactivity is present throughout the cytoplasm of immunoreactive neurons and that most of the immunoreactivity is associated with the basal ganglia. Within the caudatoputamen (CP), nucleus accumbens, olfactory tubercle, bed nucleus of the stria terminalis, and portions of the amygdaloid complex, ARPP-21 is present in neuronal somata and dendrites. In brain regions known to receive projections from these nuclei, immunoreactivity is present in puncta (presumed axons and axon terminals). These regions include the globus pallidus, ventral pallidum, entopeduncular nucleus, lateral preoptic area, and substantia nigra.

Within the basal ganglia, ARPP-21 immunoreactivity is most intense in the olfactory tubercle, nucleus accumbens, medial portion of the $C P$, and the ventral retrochiasmatic pocket of the CP. These same areas comprise the limbic striatum, and ARPP-21 is the first substance found to be specifically enriched therein. The possibility is discussed that ARPP-21 mediates effects of multiple first messengers, including dopamine and vasoactive intestinal polypeptide, that act through cAMP.
\end{abstract}

ARPP-21 is an $a$ denosine $3^{\prime}: 5^{\prime}$-monophosphate-regulated $p$ hosphoprotein with a molecular weight of 21,000 , that is enriched in the basal ganglia (Walaas et al., 1983a). Several neurotransmitters that are capable of increasing intracellular concentrations of cAMP are also present in the basal ganglia. One of these, vasoactive intestinal polypeptide (VIP) (Sims et al., 1980; Vanderhaeghen et al., 1980), has recently been shown to regulate ARPP-21 phosphorylation in tissue cultures of striatal cells (Shalaby et al., 1987). Dopamine is also enriched in the basal ganglia and, presumably, also regulates ARPP-21 phosphorylation, via activation of the $\mathrm{D}-1$ receptor (the dopamine receptor coupled to adenylate cyclase) (see Kebabian and Calne, 1979),

\footnotetext{
Received Feb. 26, 1988; revised July 15, 1988; accepted July 18, 1988.

This research was supported by USPHS Grant MH 40899. We thank Dr. JeanAntoine Girault for testing the specificity of our monoclonal antibodies by immunoblotting, and we gratefully acknowledge the skilful technical assistance of Ms. Vicki Wells.

Correspondence should be addressed to Paul Greengard, Box 296, The Rockefeller University, 1230 York Avenue, New York, NY 10021.

a Present address: Department of Psychology, Florida State University, Tallahassee, FL 32306.

'Present address: Department of Anaesthesia, Massachusetts General Hospital, Boston, MA 02114

Copyright (C) 1989 Society for Neuroscience $0270-6474 / 89 / 030865-11 \$ 02.00 / 0$
}

as it does that of another phosphoprotein called DARPP-32 (Walaas et al., 1983b; Walaas and Greengard, 1984). Thus, the possibility exists that ARPP-21 is a third messenger that mediates the actions of multiple first messengers.

The present study was undertaken to determine the cellular and regional distribution of ARPP-21. The distribution pattern obtained is considered in relation to cytoarchitecture, hodology, and patterns of distribution of neurotransmitters and peptides. The purification and biochemical characterization of ARPP-21 are reported in the accompanying paper (Hemmings and Greengard, 1989).

\section{Materials and Methods}

Preparation of anti-ARPP-21 antibodies. Anti-ARPP-21 monoclonal antibodies 21-3D and 21-6A were prepared and characterized as described in the preceding paper (Hemmings and Greengard, 1989). These monoclonal antibodies were further purified by chromatography on DEAE Aff-Gel Blue (Bio-Rad) by the method described by the manufacturer. The immunoglobulin subtype of both preparations of monoclonal antibody was determined to be IgG-1 by Ochterlony immunodiffusion (Miles antibody subtype kit, Miles Laboratories). Aliquots of these purified monoclonal antibodies were biotinylated using N-biotinyl- $\omega$-aminocaproic acid- $N$-hydroxysuccinamide ester (Enzo Biochem) by the method described by the manufacturer. A mixture of monoclonal antibodies 21-3D and 21-6A was tested for specificity by immunoblotting and immunoprecipitation techniques. A single radioactive band was observed upon immunoblotting of a homogenate of rat caudatoputamen (CP) (Fig. 1A). Similarly, these antibodies immunoprecipitated a single phosphoprotein of $M_{r} 21,000$ from extracts of rat caudatoputamen and neocortex (Fig. 1B), using the immunoprecipitation technique described by Hemmings and Greengard (1989); $>80 \%$ of ARPP21 present in cach sample was immunoprecipitated by this technique.

Immunocytochemical localization of ARPP-21. The 2 monoclonal antibodies (21-3D and 21-6A) monospecific for ARPP-21 were mixed together to increase the probability of labeling more than one epitope and thereby increase the intensity of staining. The staining intensity produced by the mixture was, in fact, stronger than that produced when each monoclonal antibody was used separately. The immunostaining patterns produced by the monoclonal antibodies when used separately did not differ from that produced by the mixture.

Male Sprague-Dawley rats $(150 \mathrm{gm})$ were anesthetized with chloral hydrate $(350 \mathrm{mg} / \mathrm{kg})$ and perfused, via a cannula inserted into the aorta, with $150 \mathrm{ml}$ of buffer followed by $500 \mathrm{ml}$ of one of several fixatives. For each perfusion, the buffer prerinse consisted of the same buffer that was used as a diluent for the fixative employed. The fixatives included $4 \%$ formaldehyde $/ 0.5 \%$ zinc salicylate (Mugnaini and Dahl, 1983), mix tures of formaldehyde and picric acid, mixtures of glutaraldehyde and formaldehyde, and $4 \%$ formaldehyde (freshly depolymerized from paraformaldehyde). The fixative that gave the greatest sensitivity and lowest background was $4 \%$ formaldehyde in $0.1 \mathrm{~m} \mathrm{Na}$ phosphate buffer $(\mathrm{pH}$ 7.4). Consequently, this fixative was employed for these studies.

Fixative was delivered with a peristaltic pump at a pressure of 120 $\mathrm{mm} \mathrm{Hg}$. Following postfixation in situ for $1 \mathrm{hr}$, brains were cut into slabs $3 \mathrm{~mm}$ thick, which were in turn cut into sections $50-100 \mu \mathrm{m}$ thick 


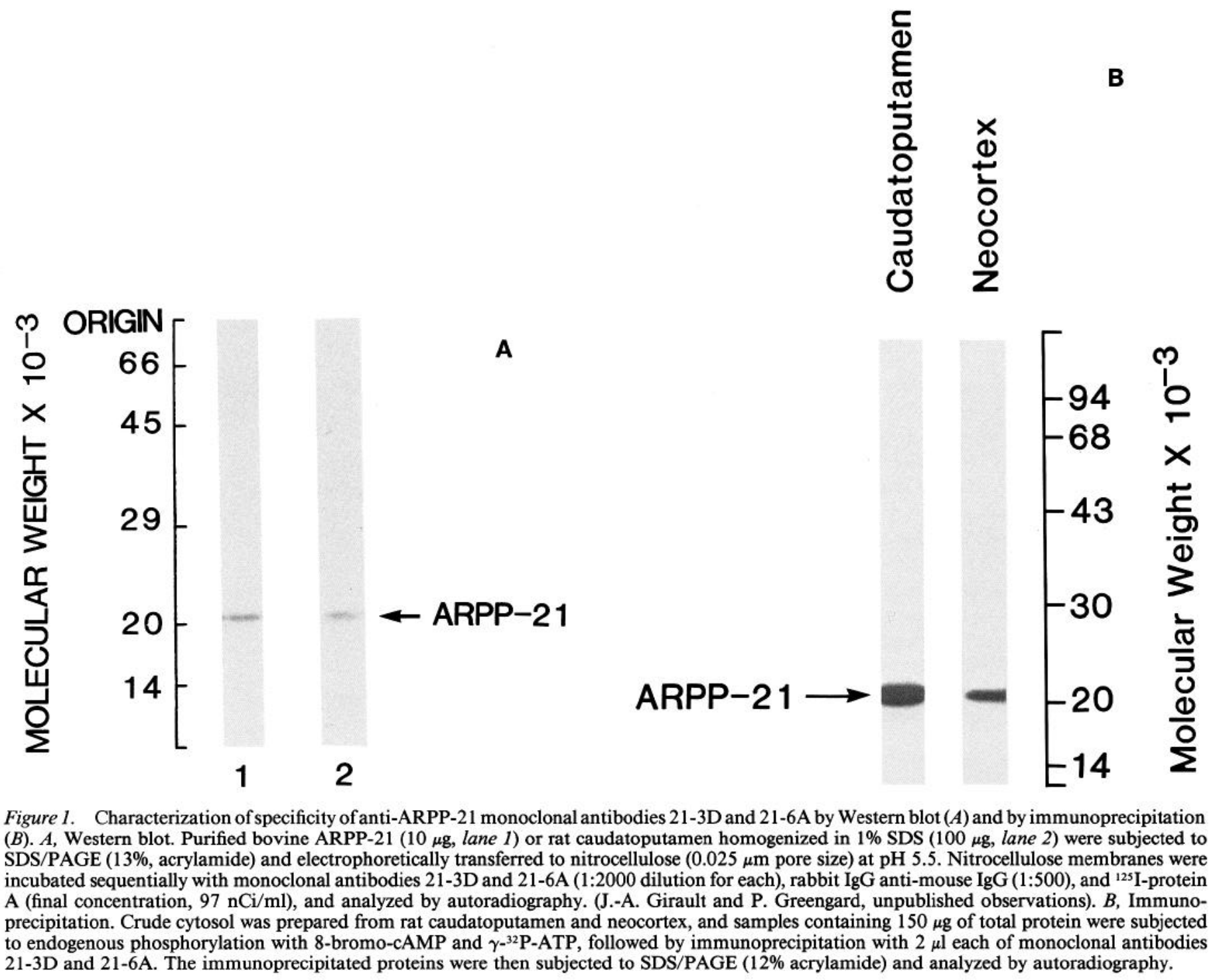

on a Vibratome. The sections were then washed $(6 \times 10 \mathrm{~min}$ in PBS: $0.01 \mathrm{~m} \mathrm{Na}$ phosphate, $0.15 \mathrm{M} \mathrm{NaCl}, \mathrm{pH} 7.4$ ), and incubated with primary antibody (diluted in PBS containing 1\% normal horse serum) overnight at room temperature on a tissue shaker. Patterns of immunostaining were assessed with primary antibody dilutions of $1: 1000$ to $1: 800,000$; the best signal-to-noise ratio was obtained with a dilution of $1: 30,000$. The remaining steps were carried out with a Vector ABC kit (Vector Laboratories) according to their instructions. Sections were incubated with biotinylated horse anti-mouse $\mathrm{IgG}(1 \mathrm{hr})$ followed by avidin-biotinHRP complex $(1 \mathrm{hr})$. PBS washes $(3 \times 10 \mathrm{~min})$ were used between steps. A penultimate wash in PBS $(3 \times 10 \mathrm{~min})$ was followed by a final

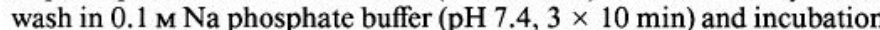
with $20 \mathrm{mg}$ of 3,3-diaminobenzidine and $10 \mu \mathrm{l}$ of $30 \%$ hydrogen peroxide in $50 \mathrm{ml}$ of $0.1 \mathrm{M} \mathrm{Na}$ phosphate buffer.

The specificity of staining protocols was verified by the substitution of the following for primary antibody: (1) diluent without primary antibody (1\% normal horse serum in PBS), (2) normal mouse serum (1: 1000), (3) monoclonal antibodies (1:500) directed against irrelevant antigens, and (4) antibodies against ARPP-21 that were preabsorbed for $1 \mathrm{hr}$ with purified ARPP-21 [0.2 mg purified ARPP- $21 / \mathrm{ml}$ of diluted anti-ARPP-21 $(1: 30,000)]$. The choroid plexus, ependymal cells, and cells in the superior olive were immunoreactive in the absence of primary antibody, and their immunoreactivity was therefore regarded as unrelated to the presence of ARPP-21. The possibility exists, however, that artifactual staining may mask bona fide staining in these regions. Omission of second antibody or of the avidin-biotin-HRP complex resulted in a total lack of staining.

\section{Results}

Immunoreactivity for ARPP-21 was observed throughout the neuronal cytoplasm, which was evenly stained. Nuclei were weakly stained or unstained (Fig. 2). Many brain regions were virtually unlabeled, while others contained either labeled neuronal somata and dendrites or labeled puncta (presumed axon terminals) and axons (Fig. 3). In some brain regions, immunostaining was very strong, and in others it was so weak that there was difficulty in distinguishing it from background staining. The relative difference between strong and weak staining can be assessed in low-power photomicrographs such as those in Figure 3, where these regions are juxtaposed within single photomicrographic fields. Brain regions that were strongly immunoreactive for ARPP-21 are discussed separately from those that were weakly immunoreactive. 

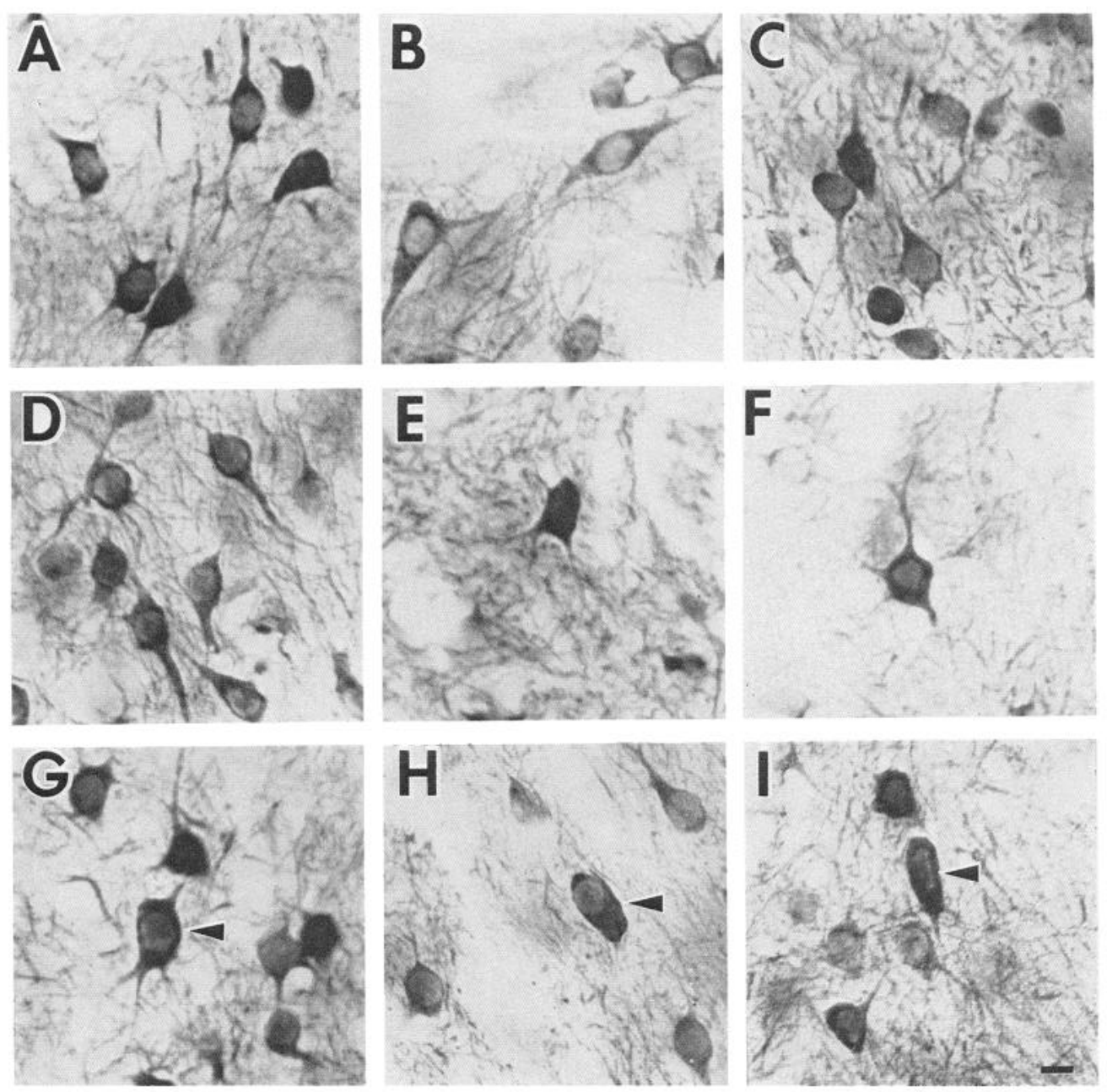

Figure 2. Photomicrographs of ARPP21-containing neurons in the caudatoputamen and nucleus accumbens. $A-F$, Typical immunoreactive neurons. These cells have somata diameters of $10-13 \mu \mathrm{m}$. $G-I$, The neurons identified by the arrowheads are somewhat larger than the typical immunoreactive somata. Such neurons were infrequently encountered. Scale bar, $10 \mu \mathrm{m}$.

\section{Telencephalon}

Strong immunoreactivity. Intensely labeled neuronal somata and dendrites were present in the olfactory tubercle, nucleus accumbens, CP and portions of the bed nucleus of the stria terminalis and amygdaloid complex. Nerve terminal staining that may have been present in these areas would be difficult to see against a background of densely packed and heavily labeled neuronal processes.

In the olfactory tubercle, somata and their dendrites were labeled in the polymorph and pyramidal layers (Fig. 4, $A, B$ ). Labeled dendrites formed a dense array of radially oriented fibers in the molecular layer. Dense immunolabeling stopped abruptly at the border between the olfactory tubercle and the piriform cortex, which was less intensely immunoreactive (Fig. $4 B$ ). The islands of Calleja, which were very weakly immunostained (Fig. $4 B$ ), were capped with small regions of strongly immunostained puncta (not shown). These regions were continuous caudally with the ventral pallidum, which also contained many labeled puncta.

Immunostaining in the nucleus accumbens was more intense than that observed in the CP (for comparison, see Fig. 6A). Within the nucleus accumbens, clusters of very heavily immunoreactive neurons were observed in all preparations (Fig. $5)$. Most of the labeled neurons corresponded in size (10-15 $\mu \mathrm{m}$ somata diameter) and number to the medium spiny neurons of the neostriatum (Fig. 5). A small number of labeled neurons were somewhat larger (18-22 $\mu \mathrm{m}$ somata diameter) (Fig. 2GI). Many neostriatal neurons were not immunoreactive, however. This was apparent in sections examined with differential interference contrast microscopy, which was used to restrict the photographic focal plane to the surface of the section, where presumably all cells were exposed to immunoreagents, and to identify the unstained figures as somata.

Overall, immunoreactivity in the $\mathrm{CP}$ was less intense and more homogeneous than that in the nucleus accumbens. Immunoreactivity was strongest in the medial portion of the $\mathrm{CP}$ in rostral sections (Fig. 6) and in both medial and lateral portions in extreme caudal sections (Fig. 7D). A cluster of densely stained cells was present in the ventral retrochiasmatic pocket of the $\mathrm{CP}$ (Figs. $6 \mathrm{~B}, 7 \mathrm{C}$ ) at a plane just rostral and dorsal to the central amygdaloid nucleus. A second cluster of strongly immunoreactive neurons was present in a location ventral to the stria terminalis and medial to the CP (Fig. $7 E$ ). This group of cells roughly corresponded to the supracapsular portion of the bed nucleus of the stria terminalis. A third cluster of intensely immunoreactive neurons formed an elongated strip along the medial border of the rostral globus pallidus (Fig. $7 F$ ). This cell group roughly corresponded to the medioposterolateral portion 


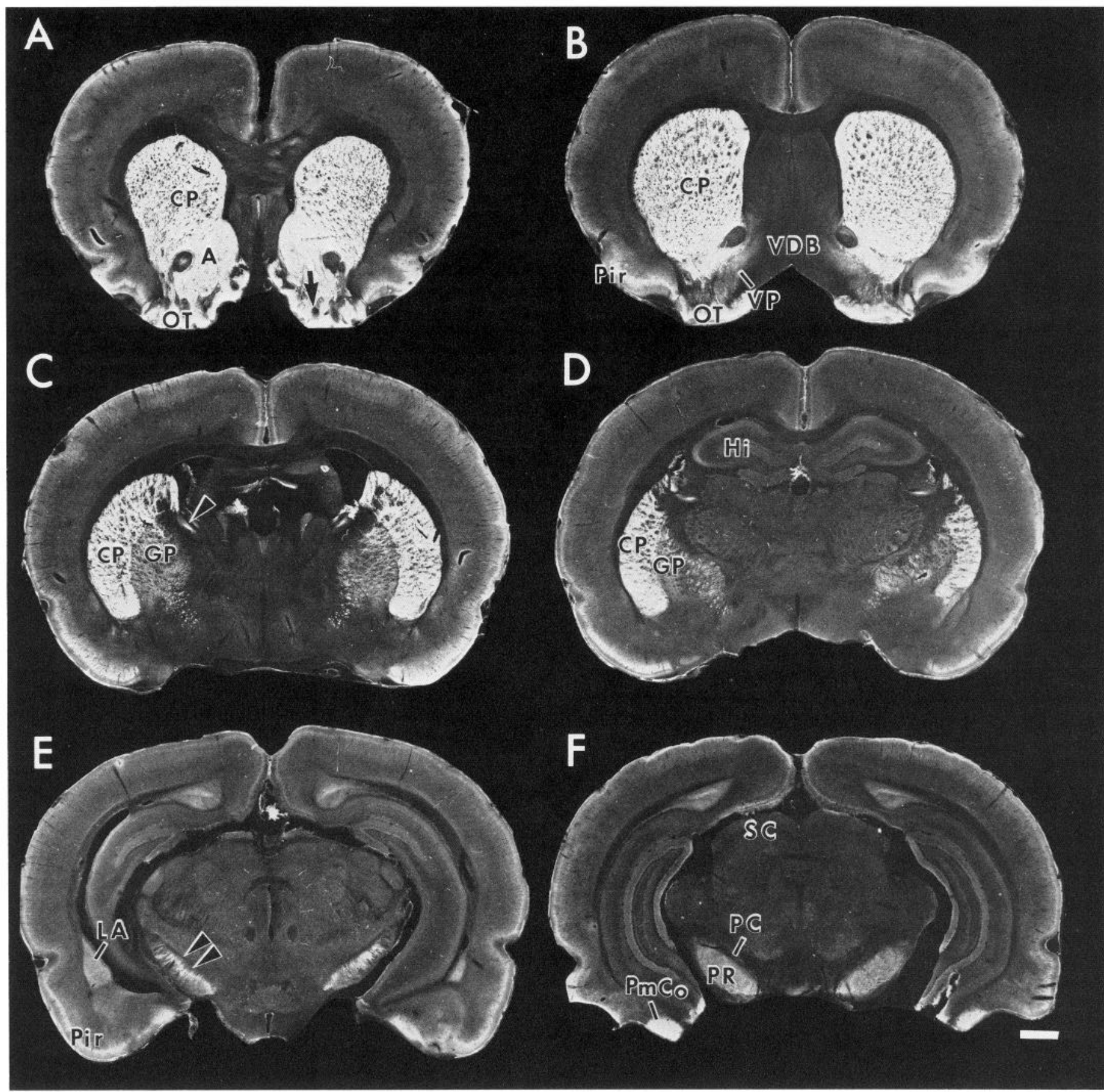

Figure 3. Photomicrographs of ARPP-21 immunostaining in coronal sections through the rat brain at the following levels: $A$, nucleus accumbens; $B$, ventral limb of diagonal band of Broca; $C$, rostral globus pallidus; $D$, caudal globus pallidus; $E$, internal capsule; and $F$, substantia nigra. These photomicrographs were prepared by placing microscope slides in an enlarger to produce negative images on photographic paper. Hence, the bright areas represent regions of immunoreactivity, and the images resemble those seen with immunofluorescence. Detail was better preserved in these negative images than after photographic conversion to positive images. Microscopy at higher magnification revealed that strongly immunoreactive somata and dendrites were present in the caudatoputamen, nucleus accumbens, olfactory tubercle, and posteromedial amygdaloid nucleus and that immunoreactive puncta (but not somata and dendrites) were present in the globus pallidus, ventral pallidum, and substantia nigra pars reticulata. Immunoreactive fibers were present in the internal capsule and stria terminalis. $A$, nucleus accumbens; $C P$, caudatoputamen; $G P$, globus pallidus; $H i$, hippocampal formation; $L A$, lateral amygdaloid nucleus; $O T$, olfactory tubercle; $P C$, pars compacta of the substantia nigra; $P i r$, piriform cortex; $P m C o$, posteromedial cortical amygdaloid nucleus; $P R$, pars reticulata of the substantia nigra; $S C$, superior colliculus; $V D B$, vertical limb of the diagonal band of Broca; $V P$, ventral pallidum; arrow, an island of Calleja; arrowhead, stria terminalis; double arrowhead, internal capsule. Scale bar, $1 \mathrm{~mm}$.

of the bed nucleus of the stria terminalis. In horizontal and sagittal sections through the neostriatum, a rostrocaudal gradient of staining was not observed.

Fibers immunoreactive for ARPP-21 were present in the anterior commissure (Fig. $4 D$ ) and stria terminalis (Fig. $3 C$ ), the internal capsule (Fig. $3 E$ ), and in its caudal continuation as the crus cerebri. Immunoreactive fibers were no longer detected in sections through the crus cerebri at the level of the substantia nigra.

Strongly immunoreactive puncta (presumed axon terminals) 

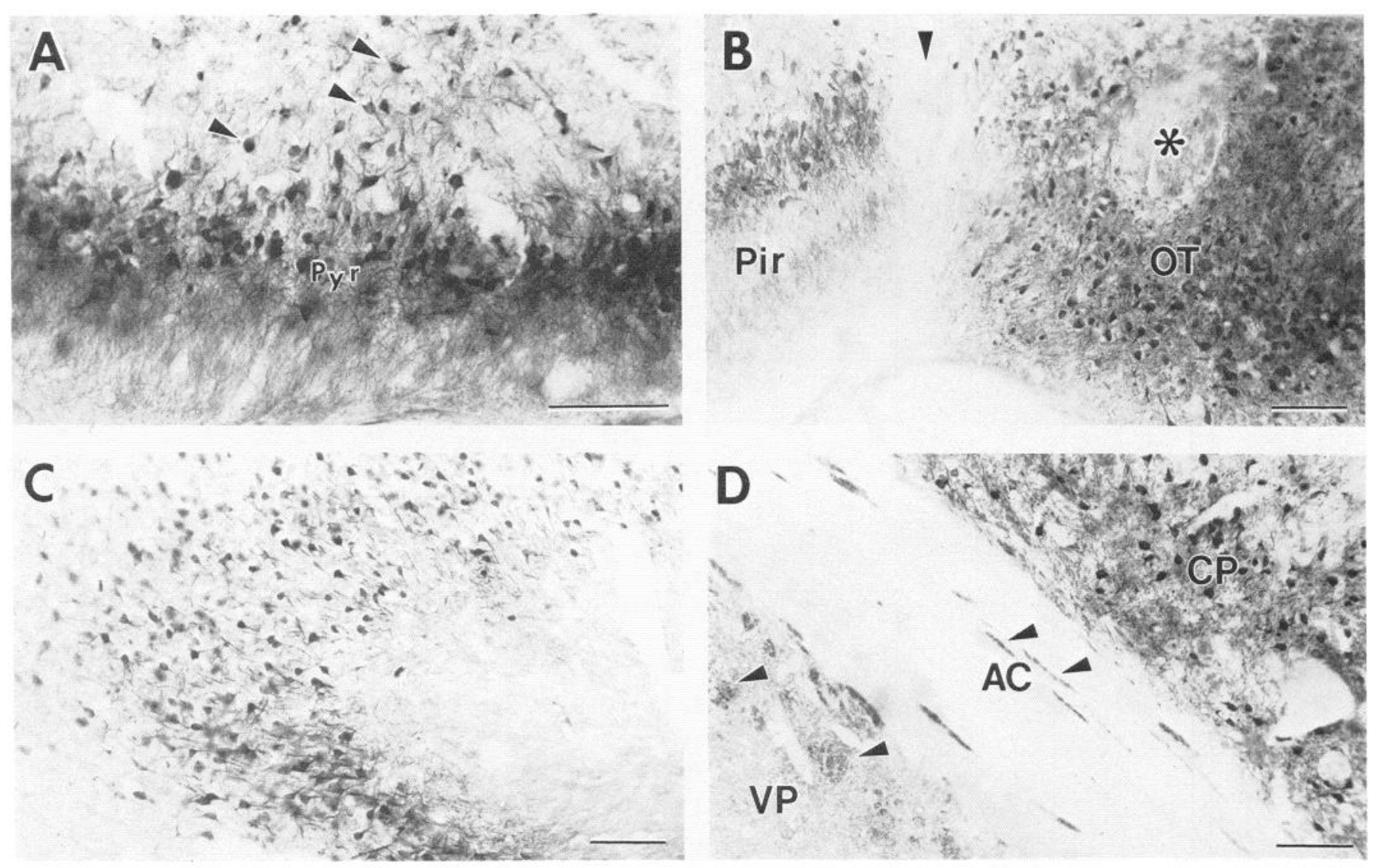

Figure 4. Photomicrographs of ARPP-21-containing cells in the basal forebrain. A, Olfactory tubercle. Neurons in the pyramidal layer (Pyr) were heavily immunoreactive, as were a smaller number of neurons (arrowheads) in the polymorph layer. Immunolabeled dendrites were present throughout the plexiform layer. B, Border between piriform cortex (Pir) and olfactory tubercle (OT). At many (but not all) rostrocaudal levels, a zone of sparse immunoreactivity separated the piriform cortex from the olfactory tubercle (arrowhead). Immunoreactivity was slightly less in the piriform cortex than in the olfactory tubercle. Weak, diffuse staining was present in an island of Calleja (asterisk). C, Perirhinal cortex. Many neurons in the superficial layers were labeled. The pial surface at the rhinal fissure was at the right edge of the photomicrograph. $D$, Caudatoputamen $(C P)$, anterior commisure $(A C)$, and ventral pallidum $(V P)$. Immunoreactive neurons were present in the $C P$. Immunoreactive fibers were present in the AC and VP (arrowheads). The VP also contained immunoreactive puncta but did not contain immunoreactive somata or dendrites. In all photomicrographs, dorsal is up. Scale bars, $100 \mu \mathrm{m}$.

were present throughout the globus pallidus (Fig. 8), entopeduncular nucleus, ventral pallidum, and substantia nigra pars reticulata. Patches of puncta that were more intensely immunoreactive than the surrounding matrix of stained puncta were present in the pallidal regions and in the substantia nigra pars reticulata. These patches appeared to be axon bundles cut in cross section and strongly resembled the immunoreactive axon bundles present in the internal capsule. Immunoreactive puncta were also present in the lateral septum, and in the medial portion of the bed nucleus of the stria terminalis; these puncta were not as densely packed as those in pallidal regions or in the substantia nigra.

In the amygdaloid complex, neurons throughout the corticomedial (Fig. 3E), posterior cortical (Fig. $3 F$ ), and cortical nuclei were heavily stained. The central nucleus contained a smaller number of moderately stained neurons. Moderately stained neurons were present throughout the lateral nucleus and along the lateral edge of the basolateral nucleus (Fig. $3 E$ ). Scattered weakly labeled neurons were present in the remainder of the basolateral nucleus.

Weak immunoreactivity. Immunoreactivity in the superficial layers of the neocortex was substantial (Fig. $9 A$ ) but less intense than that seen in the neostriatum (Fig. $3 A-D$ ). The greatest number of immunolabeled cells were present in temporal and occipital cortex, with fewer in the parietal cortex and fewer still in the frontal cortex. In frontal cortex, labeling was restricted primarily to layers I and II. In the remainder of the neocortex, the additional labeled neurons were present in layers III and IV. Dendrites from layer II neurons could be followed to layer I, where they branched profusely.

In the cingulate cortex, immunostaining was stronger than in neocortex, and moderately labeled neurons were present in layers II-III (Fig. 9B). Labeled neurons were also present in the presubiculum and parasubiculum. In the perirhinal cortex, there were a great many labeled neurons (Fig. 4C). Mitral cells and some tufted cells were weakly stained in the olfactory bulb.

\section{Diencephalon}

A cluster of strongly immunoreactive puncta (presumed axon terminals) were present in the lateral preoptic area. These puncta were not as densely packed as those in the pallidal regions. Areas of weak immunoreactivity were not observed.

\section{Mesencephalon}

No immunoreactive cell bodies were observed in the mesencephalon. Strongly immunoreactive puncta were present in the 

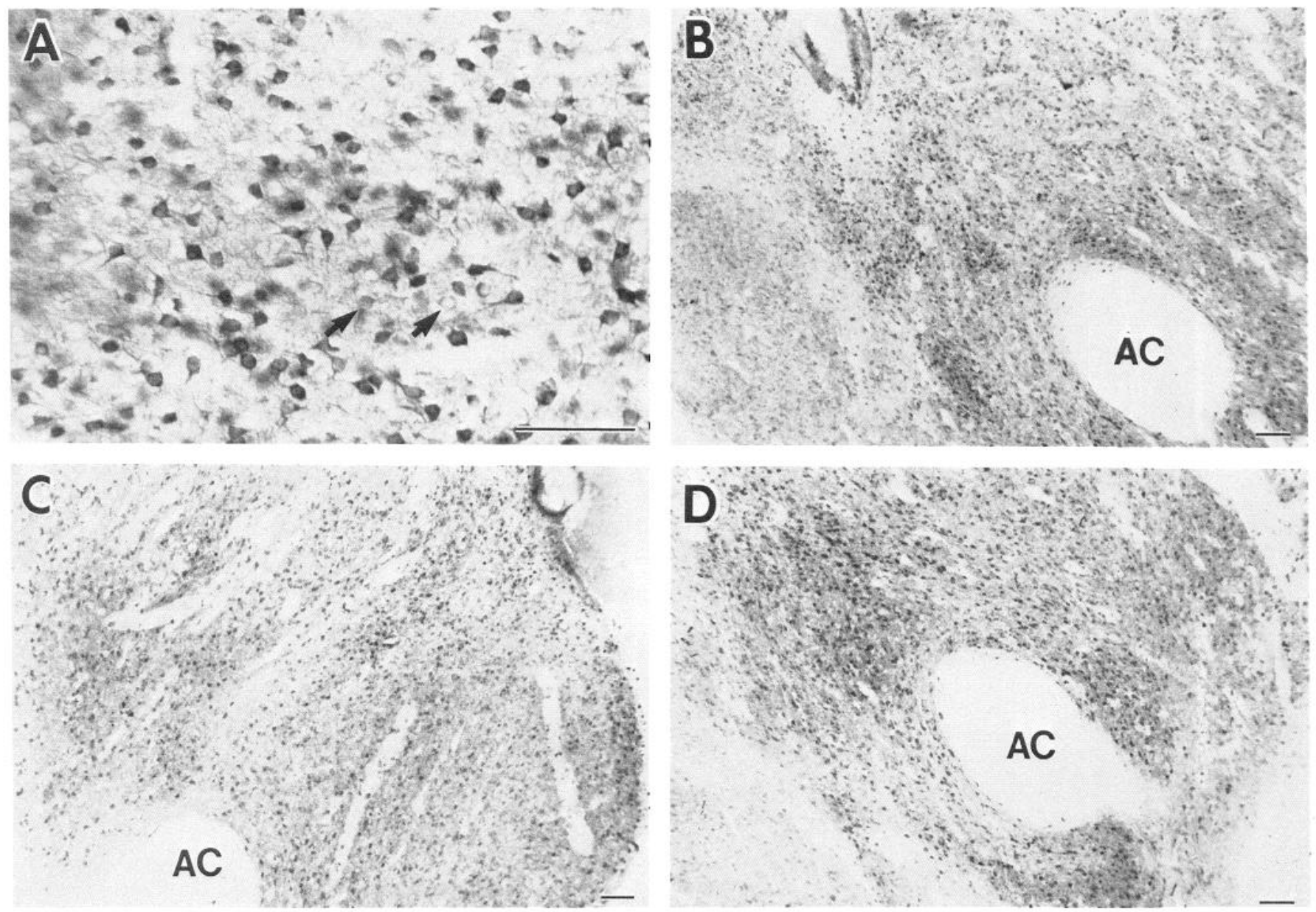

Figure 5. Photomicrographs of clusters of ARPP-21 immunoreactive neurons in nucleus accumbens. $A$, High-power photomicrograph of one cluster of heavily labeled ARPP-21-containing neurons. A small number of the neurons are lightly labeled (arrows). $B-D$, Low-power photomicrographs of ARPP-21-containing cell clusters. In $B$ and $D$ the medial edge is to the left, and in $C$ it is to the right. $A C$, anterior commissure. Scale bars, $100 \mu \mathrm{m}$

substantia nigra. These puncta were distributed primarily in the pars reticulata, but a small number were also present in the pars compacta. Some of the puncta formed strongly immunoreactive patches resembling axon bundles. Other puncta surrounded somata and dendrites (Fig. 10) and resembled axon terminals. A
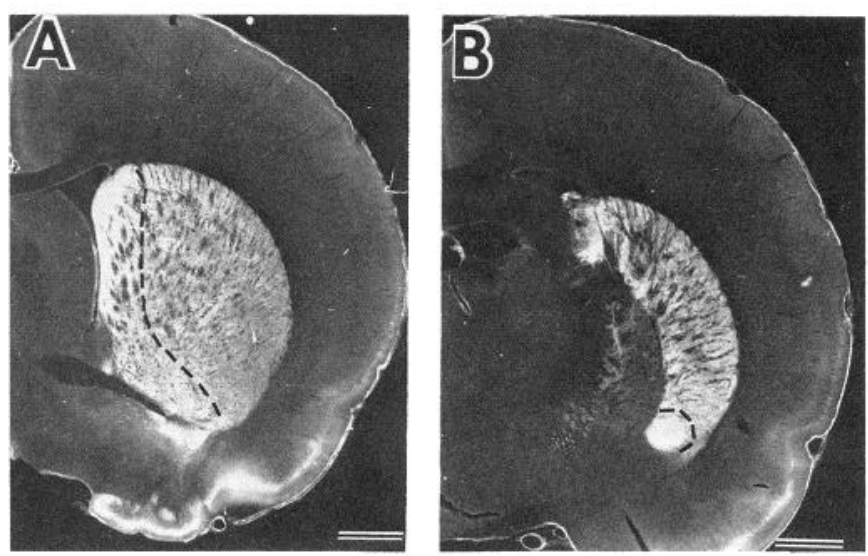

Figure 6. Low-power photomicrographs exposed to show the gradient of ARPP-21 staining in the caudatoputamen. ARPP-21 immunoreactivity was greater in the nucleus accumbens and along the medial edge of the caudatoputamen outlined by the dashed line in $A$ and in the ventral retrochiasmatic pocket of the caudatoputamen outlined by the dashed line in $B$. Scale bars, $1 \mathrm{~mm}$. small number of immunoreactive puncta were also present in the ventral tegmental area.

\section{Metencephalon, myelencephalon, and spinal cord}

No bona fide immunoreactivity for ARPP-21 was observed in these subdivisions.

\section{Discussion}

ARPP-21 immunoreactivity appears to be uniformly distributed throughout the neuronal cytoplasm, an observation consistent with biochemical studies showing that ARPP- 21 is a cytosolic protein (Hemmings and Greengard, 1989). Also consistent with previous studies (Walaas et al., 1983a) is the observation that ARPP-21 immunoreactivity is enriched in the basal ganglia and substantia nigra. ARPP-21-containing somata and dendrites are present in the $\mathrm{CP}$, nucleus accumbens, and olfactory tubercle, lateral portion of the bed nucleus of the stria terminalis, and portions of the amygdaloid complex. In certain other brain regions, immunoreactivity is present in puncta, which, in studies at the electron microscopic level, are resolved as nerve terminals and axons (Ouimet and Greengard, unpublished observations). These areas include the globus pallidus, entopeduncular nucleus, ventral pallidum, substantia nigra pars reticulata, and lateral preoptic area. Our interpretation of this pattern of nerve terminal staining is that it represents the terminal fields 

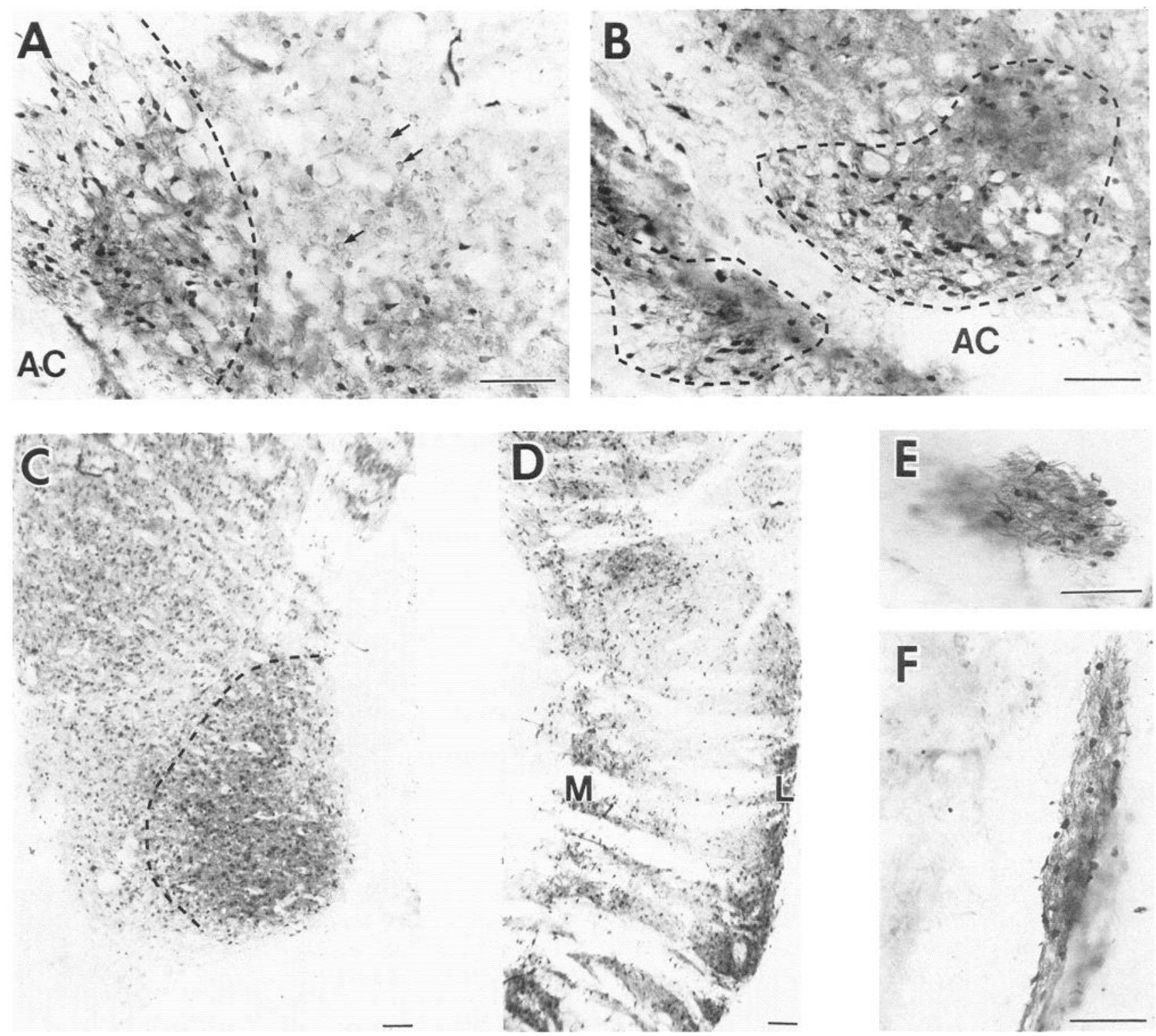

Figure 7. Photomicrographs of clusters of ARPP-21-immunoreactive neurons in caudatoputamen. $A$, Cluster of strongly immunoreactive cells (dashed line) dorsal to the medial portion of the posterolateral limit of the anterior commissure $(A C)$. The cells in this area are more intensely stained than those in adjacent areas (see, for example, arrows). B. Clusters of strongly immunoreactive cells (dashed lines) dorsal and ventral to the lateral portion of the posterior limb of the anterior commissure $(A C)$. $C$, A cluster of immunoreactive cells in the ventral retrochiasmatic pocket of the caudatoputamen (dashed line). Compare with lower-power photomicrograph in Figure $6 B$. $D$, The caudal portion of the caudatoputamen showing heavily immunoreactive neurons along the medial $(M)$ and lateral $(L)$ edges and sparse labeling in the center. $E$, A cluster of strongly immunoreactive cells located medial to the stria terminalis and just ventral to the lateral edge of the fimbria. This cluster represents the supracapsular portion of the bed nucleus of the stria terminalis. $F$, A cluster of strongly immunoreactive cells medial to the globus pallidus. This cluster may represent a portion of the medial posterolateral bed nucleus of the stria terminalis. Scale bars, $100 \mu \mathrm{m}$.

arising from distant ARPP-21-positive somata, an interpretation consistent with known anatomical relationships. Thus, the CP projects to the globus pallidus (Voneida, 1960; Szabo, 1962, 1970; Cowan and Powell, 1966; Nagy et al., 1978; Tulloch et al., 1978; Graybiel et al., 1979), to the entopeduncular nucleus (Adinolfi, 1969; Nagy et al., 1978), and to the substantia nigra (Voneida, 1960; Niimi et al., 1970; Szabo, 1970; Grofova, 1975; Bunney and Aghajanian, 1976; Nagy et al., 1978; Tulloch et al., 1978); ARPP-21-containing neurons in the CP are the probable source of ARPP-21-immunoreactive axons and terminals in those regions. Likewise, the nucleus accumbens projects to the ventral pallidum and to the dorsomedial portion of the substantia nigra (Swanson and Cowan, 1975; Conrad and Pfaff,
1976; Nauta et al., 1978; Phillipson, 1978, 1979); ARPP-21containing somata in the nucleus accumbens are the probable source of labeled terminals in those areas. The amygdaloid complex projects to the lateral preoptic area (Heimer and Nauta, 1969); ARPP-21 cells in the amygdaloid complex are the probable source of terminals in that region.

\section{Does the ARPP-21 staining pattern correlate with identifiable anatomical systems?}

Immunoreactivity within the basal ganglia is not homogeneous, and there is a striking correlation between the regions most heavily immunoreactive for ARPP-21 and the portions of the $\mathrm{CP}$ and nucleus accumbens that belong to the limbic striatum 


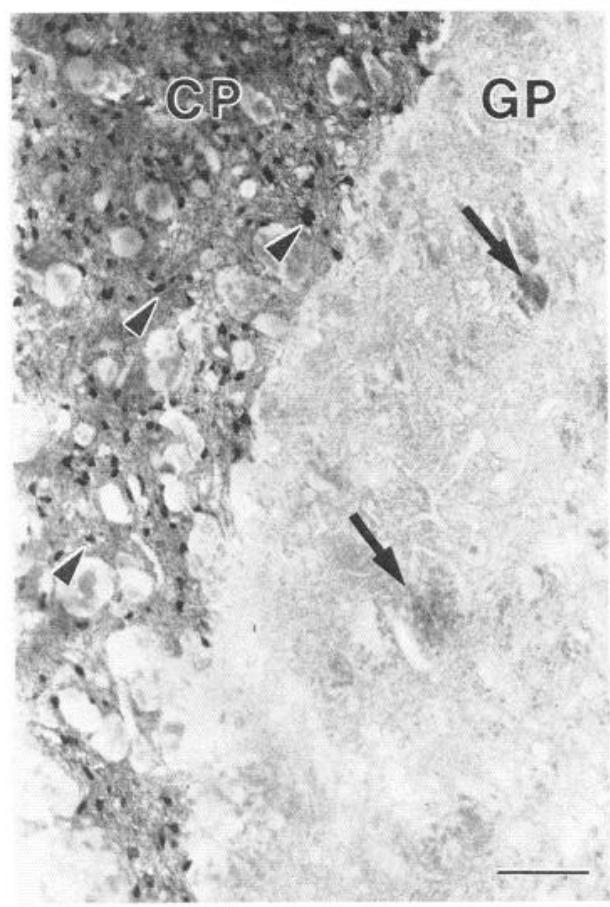

Figure 8. Photomicrograph of a coronal section through the caudatoputamen $(C P)$ and globus pallidus $(G P)$. Immunoreactive somata (see arrowheads, for examples) were present in the CP but absent from the GP. The GP contained many immunoreactive puncta, some of which were strongly stained and gathered into fascicles resembling axon bundles (arrows). Scale bar, $100 \mu \mathrm{m}$.

as defined by Nauta (1982). The limbic striatum receives a convergent input from the prefrontal cortex, ventral tegmental area, and amygdaloid complex, and consists largely of the olfactory tubercle, nucleus accumbens, a medial portion of the $\mathrm{CP}$, and a ventral retrochiasmatic pocket of the $\mathrm{CP}$ lying just dorsal to the central nucleus of the amygdaloid complex (Nauta, 1982). In each of these limbic striatal regions, immunoreactivity for ARPP-21 is very strong.

\section{Is the ARPP-21 colocalized with a specific type of neurotransmitter system?}

Many neurotransmitters and related substances, including GABA, glutamic acid decarboxylase, substance P, enkephalin, and somatostatin have been associated with medium-size neu-

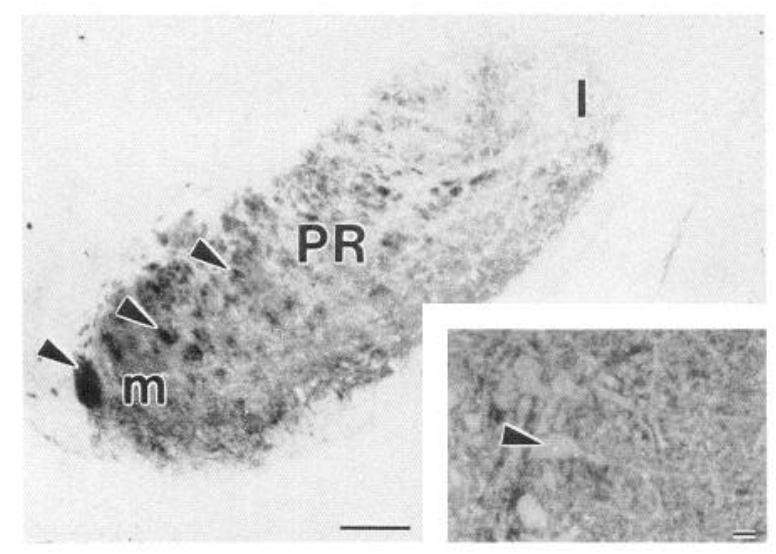

Figure 10. Photomicrographs of the substantia nigra pars reticulata. The pars reticulata $(P R)$ contained a great number of fine immunoreactive puncta. Bundles of immunoreactive axons were heavily stained (arrowheads). $m$, medial; $l$, lateral. Scale bar, $1 \mathrm{~mm}$. Inset, Higher-power photomicrograph of the PR. Arrowhead points to an unlabeled neuron surrounded by fine puncta. Scale bar, $10 \mu \mathrm{m}$.

rons in the CP (for review, see Graybiel and Ragsdale, 1983). Thus, the possibility exists that ARPP-21 colocalizes with one or another of these substances. In fact, the enkephalinergic neurons of the rat CP project primarily to the globus pallidus (Del Fiacco et al., 1982; Haber and Nauta, 1983), and this projection also contains ARPP-21. Similarly, the GABAergic and substance P-containing cells project from the CP to the entopeduncular nucleus (Brownstein et al., 1977; Hong et al., 1977; Kanagawa et al., 1977; Jessell et al., 1978; Haber and Nauta, 1983) and substantia nigra (Jessell et al., 1978), and this projection also contains ARPP-21. Moreover, ARPP-21 is present in some neurons in the CP that were somewhat larger than typical medium-sized spiny neurons and may therefore colocalize with $\mathrm{ACh}$, which is present in large neurons (Kimura et al., 1980).

On the other hand, it can be stated unequivocally that ARPP21 is not present in all GABAergic neurons, all substance P-containing neurons, or all cholinergic neurons. Neurons in the nucleus reticularis thalami, most of which are GABAergic (Houser et al., 1980), do not contain detectable levels of ARPP-21. Similarly, neurons in the habenular nuclei are enriched in substance P (Hökfelt et al., 1975; Mroz et al., 1977) but not in ARPP-21. Neurons in the diagonal band of Broca are enriched in $\mathrm{ACh}$ but
Figure 9. A streaming potential determination with triethanolammonium as the permeant cation through $\mathrm{AChR}$ channels and with methanesulfonate as the anion. The cell was current-clamped to zero current and three $4 \mathrm{pA}$, constant-current pulses indicate the resistance. The arrows indicate when the external solution was changed. Triethanolammonium and ACh were applied in the 2 control records. The controls bracket application of the same solution containing 0.5 osmolal impermeant sucrose, which produced the osmotic pressure gradient. The streaming potential was taken as the difference between the controls and the test at early times (between about 0.1 and $0.5 \mathrm{sec}$ ).
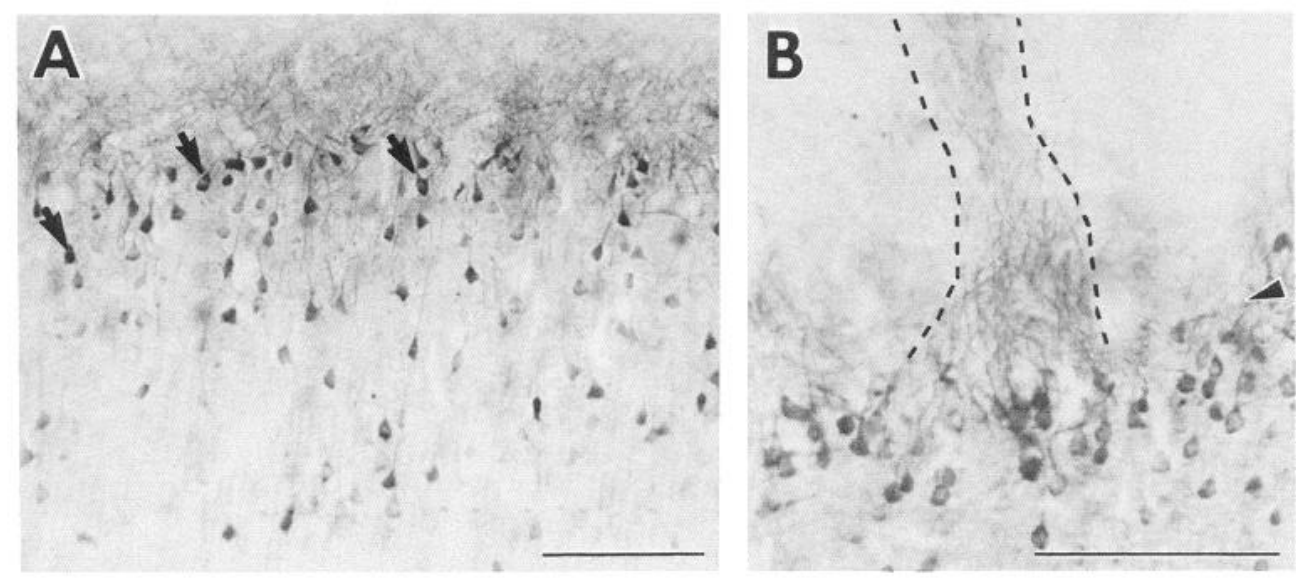
not in ARPP-21. In addition, ARPP-21 was not found within neurons of any of the monoaminergic nuclei. Hence, the staining pattern for ARPP-21 does not provide evidence for colocalization with a single type of neurotransmitter.

\section{Is ARPP-21 present in neurons innervated by a specific type of neurotransmitter system?}

It has been shown that the phosphorylation of ARPP-21 is regulated by cAMP-dependent protein kinase (Walaas et al., 1983a; Shalaby et al., 1987; Hemmings and Greengard, 1989). The first messengers that regulate ARPP-21 phosphorylation must therefore be capable of acting upon a receptor to generate cAMP. Dopamine is a reasonable candidate for such a first messenger because it works through the D-1 dopamine receptor to generate cAMP (Kebabian and Calne, 1979), and all of the strongly immunoreactive ARPP-21 neurons lie within the terminal projection fields of dopaminergic neurons. In fact, ARPP21 is present in a great number of neurons projecting from the $\mathrm{CP}$ and nucleus accumbens to the pallidum and substantia nigra and such projection neurons have been shown to contain the D-1 dopamine receptor (McGeer et al., 1976; Schwarcz and Coyle, 1977; Cross and Waddington, 1981). Hence, if dopamine acts through a D-1 dopamine receptor to generate cAMP in ARPP-21-containing neurons, it is likely that dopamine regulates ARPP-21 phosphorylation.

In addition, recent evidence indicates that VIP, which stimulates cyclic AMP production through specific membrane receptors (Quick et al., 1978), regulates the phosphorylation of ARPP-21 in cultured striatal cells (Shalaby et al., 1987). In ccrtain brain regions, such as layer II of the neocortex, nucleus accumbens, medial CP, olfactory bulb, anterior olfactory nucleus, olfactory tubercle, and hed nucleus of the stria terminalis, there is an overlap in the distribution of VIP-immunoreactive processes (Fuxe et al., 1977; Loren et al., 1979b; Roberts et al., 1980b; Sims et al., 1980) and ARPP-21-immunopositive neurons. Other areas, such as the suprachiasmatic nuclei, contain high levels of VIP (Sims et al., 1980; Card et al., 1981; Stopa et al., 1984) but little or no ARPP-21. Possibly the target cells in such regions contain VIP receptors coupled to signal transduction systems other than adenylate cyclase.

In the nucleus accumbens, there is a similarity between the distribution of cholecystokinin (CCK)-containing nerve terminals, which originate from the ventral tegmental area (Hökfelt et al., 1980; Vanderhaeghen et al., 1980), and patches of very strong ARPP-21 immunoreactivity. In addition, both CCK (Innis et al., 1979; Larsson and Rehfeld, 1979; Loren et al., 1979a; Hendry et al., 1982; Morrison and Magistretti, 1983) and norepinephrine (Fuxe et al., 1968; for recent review, see Lindvall and Bjorklund, 1984) are enriched in the superficial layers of the neocortex, as is ARPP-21. As is the case for VIP, however, there are many brain regions that contain $\mathrm{CCK}$ or norepinephrine but not ARPP-21, and vice versa. It therefore seems likely that ARPP-21 phosphorylation is regulated by multiple first messengers that regulate cyclic AMP levels.

\section{$A R P P-21$ and DARPP-32}

DARPP-32 is a dopamine- and cyclic AMP-regulated phosphoprotein with an apparent molecular weight of $32 \mathrm{kDa}$ (Walaas et al., 1983b; Hemmings et al., 1984). The distribution of DARPP-32 (Ouimet et al., 1984; Walaas and Greengard, 1984) is remarkably similar to that of ARPP-21, although the 2 phosphoproteins are not similar biochemically (Hemmings et al.,
1984; Hemmings and Greengard, 1989). In the CP and nucleus accumbens, the great majority of medium-sized neurons are immunoreactive for ARPP-21 and DARPP-32 (Ouimet et al., 1984). This strongly suggests that ARPP-21 and DARPP-32 are colocalized in many of these neurons. On the other hand, there are apparently fewer neurons immunoreactive for ARPP-21 than for DARPP-32 in the CP (unpublished observations), raising the possibility that some medium-sized neurons contain DARPP32 but not ARPP-21. Conversely, ARPP-21 is present in some neurons with somata diameters of 18-22 $\mu \mathrm{m}$, and DARPP-32 has not been observed in neurons this large (Ouimet et al., 1984). Because ARPP-21 and DARPP-32 are both present in the majority of medium-sized neurons in the CP and nucleus accumbens, they appear to provide an example of multiple phosphoprotein substrates through which a first messenger such as dopamine, acting on a single receptor type (in this case, the D-1 dopamine receptor), can alter the physiology and/or metabolism of a target neuron.

Adenylate cyclase, like ARPP-21 and DARPP-32, is highly enriched in the basal ganglia (for reviews, see Iversen, 1975, 1977; Miller and McDermed, 1979). Moreover, there is a striking similarity when the distributions of the 2 phosphoproteins are compared with that of adenylate cyclase anatomically (Worley et al., 1986). Forskolin binding, used as a marker for adenylate cyclase, is most intense in the CP, nucleus accumbens, olfactory tubercle, and the striatal projection to the substantia nigra (Worley et al., 1986). The enrichment of ARPP-21, DARPP-32, and adenylate cyclase in the neostriatum may be related to the high concentration of dopaminergic fibers in this region.

\section{Concluding remarks}

This study demonstrates the presence of ARPP-21 in neurons of the neostriatum and in the projections of these neurons to the globus pallidus, entopeduncular nucleus, ventral pallidum, and substantia nigra. Within the basal ganglia, ARPP-21 is especially enriched within those regions defined as the limbic striatum (Nauta, 1982), and is the first substance to be described with this characteristic.

We anticipate that ARPP-21 immunocytochemistry will add to our knowledge of the neuroanatomy of the basal ganglia in general and of the limbic striatum in particular. This will be especially true if the functional role of ARPP-21 and the first messenger(s) it subserves can be established.

\section{References}

Adinolfi, A. M. (1969) Degenerative changes in the entopeduncular nucleus following lesions of the caudate nucleus: An electron microscopic study. Exp. Neurol. 25: 246-254.

Brownstein, M. J., E. A. Mroz, M. L. Tappaz, and S. E. Leeman (1977) On the origin of substance $P$ and glutamic acid decarboxylase (GAD) in the substantia nigra. Brain Res. 135: 315-323.

Bunney, B. S., and Aghajanian, G. K. (1976) The precise localization of nigral afferents in the rat as determined by a retrograde tracing technique. Brain Res. 117: 423-435.

Card, J. P., N. Brecha, H. J. Karten, and R. Y. Moore (1981) Immunocytochemical localization of vasoactive intestinal polypeptidecontaining cells and processes in the suprachiasmatic nucleus of the rat: Light and electron microscopic analysis. J. Neurosci. 1: 12891303.

Conrad, L. C. A., and D. W. Pfaff (1976) Autoradiographic tracing of nucleus accumbens efferents in the rat. Brain Res. 113: 589-596.

Cowan, W. M., and T. P. S. Powell (1966) Strio-pallidal projection in the monkey. J. Neurol. Neurosurg. Psychiatry 29: 426-439.

Cross, A. J., and J. L. Waddington (1981) Kainic acid lesions dissociate 
${ }^{3} \mathrm{H}$ spiperone and ${ }^{3} \mathrm{H}$ cis-flupenthixol binding sites in rat striatum. Eur. J. Pharmacol. 71: 327-332.

Del Fiacco, M., G. Paxinos, and A. C. Cuello (1982) Neostriatal enkephalin-immunoreactive neurons project to the globus pallidus. Brain Res. 231: 1-17.

Fuxe, K., B. Hamburger, and T. Hökfelt (1968) Distribution of noradrenaline nerve terminals in cortical areas of the rat. Brain Res. 8 : 125-131.

Fuxe, K., T. Hökfelt, S. I. Said, and V. Mutt (1977) Vasoactive polypeptide and the nervous system: Immunohistochemical evidence for localization in central and peripheral neurons, particularly intracortical neurons of the cerebral cortex. Neurosci. Lett. 5: 241-246.

Graybiel, A. M., and C. W. Ragsdale, Jr. (1983) Biochemical anatomy of the striatum. In Chemical Neuroanatomy, P. C. Emson, ed., pp. 427-504, Raven, New York.

Graybiel, A. M., C. W. Ragsdale, and S. M. Edley (1979) Compartments in the striatum of the cat observed by retrograde cell labeling. Exp. Brain Res. 34: 189-195.

Grofova, 1. (1975) The identification of striatal and pallidal ncurons projecting to the substantia nigra. An experimental study by means of retrograde axonal transport of horseradish peroxidase. Brain Res. 91: 286-291.

Haber, S., and W. J. H. Nauta (1983) Ramifications of the globus pallidus in the rat as indicated by patterns of immunohistochemistry. Neuroscience 9: 245-260.

Heimer, L., and W. J. H. Nauta (1969) The hypothalamic distribution of the stria terminalis in the rat. Brain Res. 13: 284-297.

Hemmings, H. C., Jr., and P. Greengard (1989) ARPP-21, a cyclic AMP-regulated phosphoprotein enriched in dopamine-innervated brain regions. I. Purification and characterization of the protein from bovine caudate nucleus. J. Neurosci. 9: 849-862.

Hemmings, H. C., Jr., A. C. Nairn, D. W. Aswad, and P. Greengard (1984) DARPP-32, a dopaminc- and adcnosinc $3^{\prime}: 5^{\prime}$-monophosphate-regulated phosphoprotein enriched in dopamine-innervated brain regions. II. Purification and characterization of the phosphoprotein from bovine caudate nucleus. J. Neurosci. 4: 99-1 10.

Hendry, S. M. C., K. L. Valentino, E. G. Jones, and M. C. Beinfeld (1982) An investigation of neurons displaying cholecystokinin-like immunoreactivity in the cerebral cortex of monkey and rat. Soc. Neurosci. Abstr. 8: 585.

Hökfelt, T., J.-O. Kellerth, G. Nilsson, and B. Pernow (1975) Experimental immunohistochemical studies on the localization and distribution of substance $\mathrm{P}$ in cat primary sensory neurons. Brain Res. 100: 235-252.

Hökfelt, T., L. Skirboll, J. F. Rehfeld, M. Goldstein, K. Markey, and O. Dann (1980) A subpopulation of mesencephalic dopamine neurons projecting to limbic areas contains a cholecystokinin-like peptide. Evidence from immunohistochemistry combined with retrograde tracing. Neuroscience 5: 2093-2124.

Hong, J. S., H.-Y. T. Yang, G. Racagni, and E. Costa (1977) Projections of substance $\mathbf{P}$ containing neurons from neostriatum to substantia nigra. Brain Res. 122: 541-544.

Houser, C. R., J. E. Vaughn, R. P. Barber, and E. Roberto (1980) GABA neurons are the major cell type of the nucleus reticularis thalami. Brain Res. 200: 341-344.

Innis, R. B., I. M. A. Correa, G. R. Uhl, B. Schneider, and S. M. Snyder (1979) Cholecystokinin octapeptide-like immunoreactivity: Histochemical localization in rat brain. Proc. Natl. Acad. Sci. USA 76 . 521-525.

Iversen, L. L. (1975) Dopamine receptors in the brain. Science 188: $1084-1089$

Iversen, L. L. (1977) Catecholamine-sensitive adenylate cyclases in nervous tissue. J. Neurochem. 29: 5-12.

Jessell, T. M., P. C. Emson, G. Paxinos, and A. C. Cuello (1978) Topographic projections of substance-P and GABA pathways in the striato- and pallido-nigral system: A biochemical and immunohistochemical study. Brain Res. 152: 487-498.

Kanagawa, I., P. C. Emson, and A. C. Cuello (1977) Evidence for the existence of substance P-containing fibers in the striato-nigral and pallido-nigral pathways in rat brain. Brain Res. 119:447-453.

Kebabian, J. W., and D. B. Calne (1979) Multiple receptors for dopamine. Nature 277: 93-96.

Kimura, H., P. L. McGeer, J. H. Peng, and E. G. McGeer (1980) Choline acetyltransferase-containing neurons in rodent brain demonstratcd by immunocytochemistry. Science 208: 1057-1059.
Larsson, L.-I., and J. R. Rehfeld (1979) Localization and molecular heterogeneity of cholecystokinin in the central and peripheral nervous system. Brain Res. 165: 201-218.

Lindvall, O., and A. Bjorklund (1984) General organization of cortical monoamine systems. In Monoamine Innervation of Cerebral Cortex, L. Descarries, T. R. Reader, and H. H. Jasper, eds., pp. 9-70, Liss, New York.

Loren, I., J. Alumets, R. Hakanson, and F. Sundler (1979a) Distribution of gastrin and CCK-like peptides in rat brain. Histochemistry 59: 249-257.

Loren, I., P. C. Emson, J. Fahrenkrug, A. Bjorklund, J. Alumets, R. Hakanson, and F. Sundler (1979b) Distribution of vasoactive intestinal polypeptide in the rat and mouse brain. Neuroscience 4: 19531976.

McGeer, E. G., V. T. Innanen, and P. L. McGeer (1976) Evidence on the cellular localization of adenyl cyclase in the neostriatum. Brain Res. 118: 356-358.

Miller, R. J., and J. McDermed (1979) Dopamine-sensitive adenylate cyclase. In The Neurobiology of Dopamine, A. S. Horn, J. Korf, and B. H. C. Westerink, eds., pp. 159-177, Academic, London.

Morrison, J. H., and P. J. Magistretti (1983) Monoamines and peptides in cerebral cortex. Contrasting principles of cortical organization. Trends Neurosci. 6: 146-151.

Mroz, E. A., M. J. Brownstein, and S. E. Leeman (1977) Evidence for substance $\mathrm{P}$ in the striatonigral-tract. Brain Res. 125: 305-311.

Mugnaini, E., and A.-L. Dahl (1983) Zinc-aldehyde fixation for lightmicroscopic immunocytochemistry of nervous tissues. J. Histochem. Cytochem. 31: 1435-1438.

Nagy, J. I., D. A. Carter, and H. C. Fibiger (1978) Anterior striatal projections to the globus pallidus, entopeduncular nucleus and substantia nigra in the rat: The GABA connection. Brain Res. 158: 1529.

Nauta, W. J. H. (1982) Limbic innervation of the striatum. Adv. Neurol. 35: 41-47.

Nauta, W. J. H., G. P. Smith, R. L. M. Faull, and V. B. Domesick (1978) Efferent connections and nigral afferents of the nucleus accumbens septi in the rat. Neuroscience 3: 385-401.

Niimi, K., T. Ikeda, S. Kawamura, and M. Inoshita (1970) Efferent projection of the head of the caudate nucleus in the cat. Brain. Res. 21: $327-343$.

Ouimet, C. C., P. P. Miller, H. C. Hemmings, Jr., S. I. Walaas, and P. Greengard (1984) DARPP-32, a dopamine- and adenosine $3^{\prime}: 5^{\prime}-$ monophosphate-regulated phosphoprotein enriched in dopamine-innervated brain regions. III. Immunocytochemical localization. J. Neurosci. 4: 114-124.

Phillipson, O. T. (1978) Afferent projections to A10 dopaminergic neurons in the rat as shown by the retrograde transport of horseradish peroxidase. Neurosci. Lett. 9: 353-359.

Phillipson, O. T. (1979) Afferent projections to the ventral tegmental area of Tsai and intrafascicular nucleus: A horseradish peroxidase study in the rat. J. Comp. Neurol. 187: 117-144.

Quick, M., L. L. Iversen, and S. R. Bloom (1978) Effect of vasoactive intestinal peptide (VIP) and other peptides on cAMP accumulation in rat brain. Biochem. Pharmacol. 27: 2209-2213.

Roberts, G. W., P. L. Woodhams, M. G. Bryant, T. J. Crow, S. R. Bloom, and J. M. Polak (1980a) VIP in the rat brain: Evidence for a major pathway linking the amygdala and hypothalamus via the stria terminalis. Histochemistry 65: 103-119.

Roberts, G. W., T. J. Crow, and J. M. Polak (1980b) Loss of immunoreactive VIP in the bed nucleus following lesions of the stria terminalis. Brain Res. 195: 471-475.

Schwarcz, R., and J. T. Coyle (1977) Striatal lesions with kainic acid. Neurochemical characteristics. Brain Res. 127: 235-249.

Shalaby, I., J.-A. Girault, N. Rosen, and P. Greengard (1987) Regulation by cAMP and vasoactive intestinal peptide of the phosphorylation of three low molecular weight proteins in striatal cells in culture. Soc. Neurosci. Abstr., p. 900.

Sims, K. B., D. L. Hoffman, S. Said, and E. A. Zimmerman (1980) Vasoactive intestinal polypeptide (VIP) in mouse and rat brain: An immunocytochemical study. Brain Res. 186: 165-183.

Stopa, E. G., J. C. King, R. Lydic, and W. C. Schoene (1984) Human brain contains vasopressin and vasoactive intestinal polypeptide neuronal subpopulations in the suprachiasmatic region. Brain Res. 297: $159-163$.

Swanson, L. W., and W. M. Cowan (1975) $\Lambda$ note on the connections 
and development of the nucleus accumbens. Brain Res. 92: 324-330.

Szabo, J. (1962) Topical distribution of the striatum efferents in the monkey. Exp. Neurol. 5: 21-36.

Szabo, J. (1970) Projections from the body of the caudate nucleus in the rhesus monkey. Exp. Neurol. 27: 1-15.

Tulloch, I. F., G. W. Arbuthnott, and A. K. Wright (1978) Topographical organizations of the striatonigral pathway revealed by anterograde and retrograde neuroanatomical tracing techniques. J. Anat. 127: $425-441$

Vanderhaeghen, J. J., F. Lotstra, J. De Mey, and C. Gilles (1980) Immunohistochemical localization of cholecystokinin- and gastrinlike peptide in the brain and hypophysis of the rat. Proc. Natl. Acad. Sci. USA 7: 1190-1194.

Voneida, T. J. (1960) An experimental study of the course and destination of fibers arising in the head of the caudate nucleus in the cat and monkey. J. Comp. Neurol. 115: 75-88.
Walaas, S. I., and P. Greengard (1984) DARPP-32, a dopamine and adenosine $3^{\prime}: 5^{\prime}$-monophosphate regulated phosphoprotein enriched in dopamine-innervated brain regions. I. Regional and cellular distribution in the rat brain. J. Neurosci, 4: 84-98.

Walaas, S. I., A. C. Nairn, and P. Greengard (1983a) Regional distribution of calcium- and cyclic AMP-regulated protein phosphorylation systems in mammalian brain. II. Soluble systems. J. Neurosci. 3: 302-311

Walaas, S. I., D. W. Aswad, and P. Greengard (1983b) A dopamine and cyclic AMP-regulated phosphoprotein enriched in dopamineinnervated brain regions. Nature 301: 69-71.

Worley, P. F., J. M. Baraban, E. B. DeSouza, and S. H. Snyder (1986) Mapping second messenger systems in the brain: Differential localizations of adenylate cyclase and protein kinase C. Proc. Natl. Acad. Sci. USA 83: $4053-4057$. 